\title{
$\beta$-glucanos: ¿qué tipos existen y cuáles son sus beneficios en la salud?
}

\author{
$\beta$-glucans: what types exist and \\ what are their health benefits?
}

\begin{abstract}
$\beta$-glucans are glucose polymers (polysaccharides) of high molecular weight naturally present in the cell wall of various living organisms such as bacteria, yeasts, fungus and plants (mainly cereals like barley and oat). $\beta$-glucans are GRAS and are currently used as texturing agents by the food industry. Furthermore, scientific evidence suggest that, depending on their physicochemical structure and source, their intake would be associated with health promoting effect such as the decrease of plasma cholesterol and the improvement of the glycemic index of the $\beta$-glucancontaining foodstuffs. Accordingly, a health claim related with the intake of cereal $\beta$-glucans and plasma cholesterol improvement has been accepted by the FDA in U.S.A. and by the EFSA in the E.U. For this reason, $\beta$-glucans are increasingly used in the world for the development of functional foods. However, the effect of yeast $\beta$-glucans on immune system stimulation is still discussed. Key words: $\beta$-glucans, Immune function, Resistance to infections, Blood cholesterol, Blood glucose.
\end{abstract}

\section{INTRODUCCIÓN}

La importancia de la fibra dietética como parte de una dieta equilibrada para la salud del ser humano está ampliamente reconocida. Los $\beta$-glucanos son un tipo de fibra soluble para los cuales se han propuesto efectos sobre la glicemia, los niveles de insulinemia, el colesterol y la inmunidad. Cereales como la cebada y la avena son reconocidos como buenas fuentes de $\beta$-glucanos pero también se pueden encontrar en algas y hongos. Asimismo, sus propiedades parecen estar relacionadas con su peso molecular, estructura química y características reológicas, las cuales pueden variar según el origen del $\beta$-glucano (1).

Debido a sus efectos potenciales sobre la salud, la industria de alimentos ha utilizado cada vez más los $\beta$-glucanos para el desarrollo de alimentos funcionales. Además, sus propiedades reológicas han estimulado su incorporación a distintas matrices alimentarias con el objetivo de mejorar la estabilidad, textura y vida útil del alimento, en remplazo de ciertos aditivos 0 agentes texturizantes artificiales (2).

En los cereales, los $\beta$-glucanos se encuentran concentradas en las paredes celulares del endospermo, cuyo desarrollo es dependiente de las condiciones geoclimáticas; estas, por lo tanto, inciden en el contenido de $\beta$-glucano del cereal. Este es regulado por la enzima $\beta$-glucano endohidrolasa $(1 \rightarrow 3,1 \rightarrow 4)$, también conocida como licheninasa o $1 \rightarrow 3,1 \rightarrow 4 \beta$-glucanasa, cuya función es promover la degradación de la pared celular
Sebastián Pizarro C. (1)

Ana María Ronco M. (2) Martín Gotteland R. (1)

1) Departamento de Nutrición, Facultad de Medicina Universidad de Chile, Santiago, Chile (2) Laboratorio de Nutrición y Regulación Metabólica, Unidad de Nutrición Humana, INTA, Universidad de Chile. Santiago, Chile.

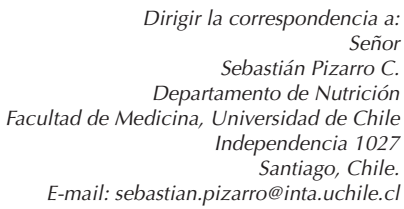

Este trabajo fue recibido el 21 de Enero de 2014 y aceptado para ser publicado el 10 de Septiembre de 2014.

del endospermo durante la germinación (3). Entre los cereales que poseen mayor contenido de $\beta$-glucanos (en g por $100 \mathrm{~g}$ de peso seco) está la cebada (2-20 g, con 65\% hidrosoluble), seguida por la avena (3-8 g, con $82 \%$ hidrosoluble). Otros cereales también lo contienen, pero en cantidades mucho menores tales como el sorgo (1.1 a $6.2 \mathrm{~g})$, el centeno (1.3 a $2.7 \mathrm{~g})$, el maíz $(0,8-1,7 \mathrm{~g})$, el triticale $(0,3-1,2 \mathrm{~g})$, el trigo $(0,5-$ $1,0 \mathrm{~g})$, y el arroz $(0,13 \mathrm{~g})$ (4). Otras fuentes de $\beta$-glucanos son algunos tipos de algas (5) y de setas como el Reishi, Shiitake y Maitake (6)

\section{CARACTERÍSTICAS FÍSICO-QUÍMICAS DE LOS $\beta$-GLUCANOS}

Los $\beta$-glucanos son homopolisacáridos lineales de glucosa unidos a través de enlaces $\beta-(1 \rightarrow 3)$ y $\beta-(1 \rightarrow 4)$ y que pueden presentar ramificaciones ( 7$)$. No son digeribles en el intestino delgado del ser humano debido a que no existen enzimas pancreáticas o intestinales capaces de degradarlas, por lo cual son clasificados como fibra dietética soluble (8). La estructura macromolecular y en particular el tipo de enlaces de la cadena principal y de sus ramificaciones dependen de la fuente del $\beta$-glucano (tabla 1) y permite diferenciarlos entre sí. Por ejemplo, los $\beta$-glucanos de avena y cebada (figura 1 A) están compuestos de cadenas no ramificadas con enlaces $\beta(1 \rightarrow 3)$ y $\beta(1 \rightarrow 4)$ mientras que los $\beta$-glucanos de levaduras y hongos (figura 1B) están compuestos de cadenas de glucosa unidos 
por enlaces $\beta(1 \rightarrow 3)$ y cadenas laterales unidas por enlaces $\beta$ $(1 \rightarrow 6)(1,9)$. Los $\beta$-glucanos de levaduras y hongos, a pesar de poseer enlaces y ramificaciones similares, difieren en la longitud de sus cadenas, siendo más largas en las levaduras. Las diferencias en el tipo de enlace y de ramificación influencian el tamaño de la molécula, su estructura terciaria, su carga eléctrica, su conformación en solución (hélice triple o simple, o espiral aleatoria) y sus propiedades de solubilidad $(1,10)$.

\section{EFECTOS DE LOS B-GLUCANOS SOBRE LA SALUD}

Los efectos de los $\beta$-glucanos en la salud dependen de su fuente de origen; a aquellos provenientes de cereales se les ha atribuido propiedades beneficiosas de tipo metabólico (reducción de las concentraciones plasmáticas de colesterol y de glucosa) y a aquellos que provienen de levaduras propiedades inmunoestimulantes.

\section{A) $\beta$-glucanos de cereales}

Las propiedades funcionales de los $\beta$-glucanos han sido atribuidas en particular al hecho que forman soluciones viscosas en solución acuosa, como ocurre en el tubo digestivo (11, 12). Esta viscosidad hace que los $\beta$-glucanos retrasen el vaciamiento gástrico e interfieran con el contacto entre las enzimas pancreáticas y sus substratos en el lumen intestinal, frenando

\section{TABLA 1}

Estructuras de $\beta$-glucanos según su fuente.

Tipo de $\beta$-glucano

Estructura

Bacteria

Hongo

Levadura

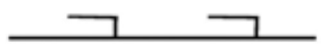

Cereal

\section{Descripción}

Glucano lineal $\beta-1,3$ (ej: Curdlan)

Glucano ramificaciones cortas $\beta-1,6$ y $\beta-1,3$ (ej: Esquizofilano)

Glucano ramificaciones largas

$\beta-1,6$ y $\beta-1,3$ (ej: $\beta$-glucano WGP, Betafectina)

Glucano lineal $\beta-1,3 / 1,4$

(ej: avena, cebada, centeno)

Adaptado y autorizado de: Volman J, Ramakers J, Plat J. Dietary modulation of immune function by $\beta$-glucans. Physiol Behav. 2008; $94(2): 276-84$.

\section{FIGURA 1}

(A): Unidades políméricas de $\beta$-(1-4)-D-glucopiranosil $\beta$-(1-3)-D-glucopiranosil. (B): Unidades poliméricas de $\beta$-(1-3)-D-glucopiranosil con ramificaciones en $\beta$-(1-6).

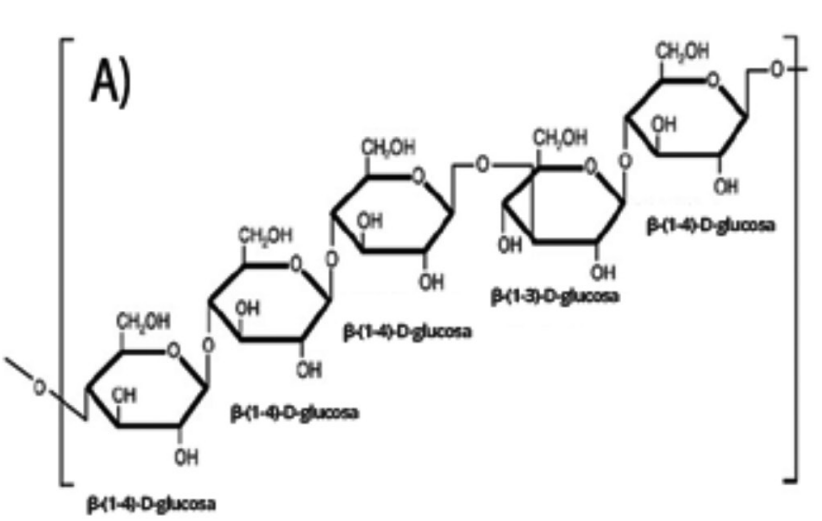

$\beta$-glucano de cereal

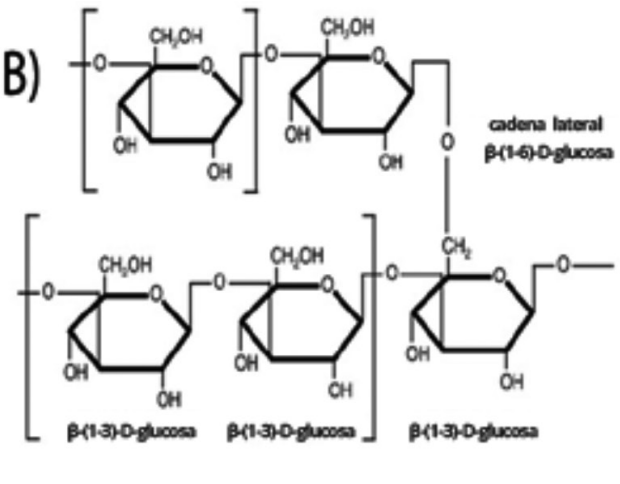

$\beta$-glucano de levadura 
los procesos de digestión y absorción de los nutrientes. Esta propiedad podría explicar el efecto de los $\beta$-glucanos sobre la reducción de las concentraciones plasmáticas de colesterol y del índice glicémico $(8,13)$.

Los estudios del efecto de $\beta$-glucanos sobre la absorción de colesterol han reportado resultados divergentes. En un estudio realizado en 62 pacientes con hipercolesterolemia moderada, la administración de $3 \mathrm{~g}$ de $\beta$-glucanos de avena o de salvado de trigo en yogurt bajo en grasa por 8 semanas no afectó los niveles de colesterol (14), mientras que Karmally et al. reportaron una disminución significativa de un $4,5 \%$ de estos niveles en 152 sujetos que consumían la misma cantidad de $\beta$-glucanos de avena por 6 semanas (15). Estas diferencias podrían deberse a la composición o al proceso de preparación del alimento administrado. Esto está ilustrado por un estudio realizado en pacientes con hiperlipidemia leve que consumieron por 2 semanas $5,9 \mathrm{~g} / \mathrm{d}$ de $\beta$-glucanos de avena en panes y galletas o en jugo. Los resultados mostraron que, comparado con el grupo control, no hubo diferencias significativas en los niveles de colesterol LDL después del consumo de panes o galletas con $\beta$-glucanos pero sí las hubo después del consumo de jugo de frutas (16). Dos meta-análisis evaluando los efectos del consumo de $\beta$-glucanos sobre el perfil lipídico del ser humano han sido publicados; el primero incorporó 10 estudios aleatorios controlados, con un rango de consumo $\beta$-glucanos de avena entre 1,1 y 7,6 g/día y con períodos de ingesta desde 18 días hasta 12 semanas. Los resultados indican una disminución promedio de $5,9 \mathrm{mg} / \mathrm{dL}$ (95\% Cl [3.3-8.4 mg/dL]) de los niveles de colesterol total en los sujetos que consumían $\beta$-glucanos. Dicho efecto era mayor cuando los niveles de colesterol iniciales eran mayores a $229 \mathrm{mg} / \mathrm{dL}$ y cuando la cantidad de $\beta$-glucanos superaban los $3 \mathrm{~g} / \mathrm{d}$ (17). Estos resultados fueron confirmados en el segundo meta-análisis que concluye que el consumo de $3 \mathrm{~g} / \mathrm{d}$ de $\beta$-glucanos de avena o cebada es suficiente para disminuir los niveles de colesterol (13). Cabe destacar que en 2010, la Agencia Europea para la Inocuidad Alimentaria (European Food Safety Agency, EFSA) aprobó un Mensaje de Salud en base a la evidencia previamente descrita, que indica que "los ß-glucanos de avena disminuyen el colesterol sanguino, lo cual reduce el riesgo de enfermedad cardiovascular"(18). La agencia agrega que dicho mensaje puede ser atribuido a los alimentos cuyo consumo provee a lo menos $3 \mathrm{~g}$ de $\beta$-glucano de avena/día, una cantidad que se considera compatible con el consumo de una dieta balanceada.

Por otra parte, varios estudios han evaluado si los $\beta$-glucanos eran capaces de controlar los niveles de glicemia, ya sea en sujetos sanos o en pacientes con diabetes. Granfeldt et al., (2007) observaron una disminución significativa de la glicemia e insulinemia post-prandial después del consumo de 4 g de $\beta$-glucanos de avena, comparado con el alimento control sin $\beta$-glucanos; no observaron cambios de estos parámetros con $3 \mathrm{~g}$ de $\beta$-glucanos (19). Otro estudio evaluó el efecto de dos tipos comerciales de $\beta$-glucanos de cebada, Glucagel y Barley Balance, incorporados a espaguetis en distintas concentraciones $(0,2,4,6,8$, y $10 \%)$, con un total de 50 gramos de hidratos de carbono disponibles por porción. El Glucagel (>75\% ß-glucano) es producido mediante extracción con agua y purificación por congelación/descongelación mientras que el Barley Balance (25\% ß-glucano) se aisla a través de un proceso en seco a partir de cebada cerosa mondada. Los ßglucanos del primero tienen un bajo peso molecular (150.000 Da) comparado con el del segundo (650.000-700.000 Da). Los resultados mostraron que la ingestión de espaguetis con
10\% Barley Balance disminuyó significativamente la glicemia post-prandial y el índice glicémico, comparado con el producto control (20). Otro estudio mostró que el consumo de 4 y $8 \mathrm{~g}$ de $\beta$-glucanos de cebada en chapati (tipo de pan consumido en India) reducía el índice glicémico en un $43 \%$ y $47 \%$, respectivamente $(I G=29$ y 30$)$, en comparación con el consumo de chapati control sin $\beta$-glucano (IG = 54) (21).

Evidencias in vitro $(22,23)$ e in vivo $(24-26)$ sugieren que los $\beta$-glucanos pueden ser usados como prebióticos (8) debido a su capacidad de promover el crecimiento de microrganismos benéficos de la microbiota intestinal como los Lactobacillus y Bifidobacterium. Un reciente ensayo clínico, aleatorizado y en doble ciego realizado en 52 adultos sanos de 39 a 70 años mostró que el consumo de 0,75 g de $\beta$-glucanos de cebada por porción de torta durante 30 días aumentaba las poblaciones fecales de bifidobacteria, comparado con los controles. Estas diferencias fueron mayormente marcadas en los sujetos de más de 50 años, siendo su consumo muy bien tolerado (27). Otro estudio evaluó el consumo diario de $3 \mathrm{~g}$ de $\beta$-glucano de cebadas en $125 \mathrm{~g}$ de pan durante 3 meses en sujetos colectomizados; se observó también una modulación de la microbiota intestinal, con una reducción significativa del recuento de coliformes totales, en comparación con el periodo basal. Asimismo, hubo un incremento significativo de los ácidos grasos volátiles (butirato y acetato) en el grupo tratado, registrándose además menores puntuaciones de distención y dolor abdominal a partir del día 30 de intervención (28).

\section{B) $\beta$-glucanos de levaduras y hongos}

Otra propiedad, principalmente atribuida a los $\beta$-glucanos provenientes de hongos/levaduras, es la modulación del sistema inmune $(29,30)$. Dicho efecto podría deberse a la capacidad de los $\beta$-glucanos de estimular receptores del sistema inmune innato presentes en la membrana de los enterocitos, de las células $M$ y de las células dendríticas, mejorando la actividad fagocítica de los macrófagos y la actividad antimicrobiana de las células mononucleares y de los neutrófilos (31). Este tipo de ß-glucanos también prevendría la promoción y progresión de ciertos tipos de cáncer, actuando en forma sinérgica con los anticuerpos monoclonales y la quimioterapia (32). Esta estimulación de la inmunidad se lograría mediante el incremento de la secreción de citoquinas pro-inflamatorias y de quimioquinas (29). El principal receptor implicado en el efecto de los $\beta$-glucanos sobre la inmunidad seria la dectina-1, a pesar de que recientemente también se haya propuesto un rol para el receptor 3 del complemento, la lactosil-ceramida, Ios TLR-2 y TLR-6 y los receptores "scavengers" $(29,31,33)$. La dectina-1, conocida en el ser humano como el receptor de $\beta$-glucanos ( $\beta G R)$, es miembro de los Receptores de Reconocimiento de Patrones (PRR) quienes cumplen un rol esencial en la respuesta inmune innata dirigida contra virus, bacterias, levaduras y hongos, contribuyendo al reconocimiento y eliminación de los patógenos $(34,35)$. Este receptor es altamente expresado en células inmunes tales como las células dendríticas, los neutrófilos, eosinófilos y monocitos así como en algunas poblaciones de células $T$ y $B$ y, en menor medida, en macrófagos y enterocitos $(31,36,37)$. La dectina-1 actúa mediante transducción de señales a través de la activación de las vías Syk y Raf-1 (31). También puede actuar en forma sinérgica con los TLR que median la producción de citoquinas pro-inflamatorias como la IL-12 y el TNF- $\alpha(34,38)$.

Lebron et al. (39) reportaron que los $\beta$-glucanos del patógeno Pneumocystis carinii así como los de $\mathrm{S}$. cerevisia, la levadura utilizada para la elaboración de pan o de cerveza, 
estimulan los macrófagos a través de la activación de NF$\kappa B$, gatillando varios eventos celulares que resultarían en la producción de citoquinas y el aumento de la fagocitosis. La hipótesis de la asociación entre el receptor de dectina-1 y los $\beta$-glucanos proviene de estudios recientes en ratones "knockout" para la dectina-1 que muestran que estos animales son más susceptibles a infecciones fúngicas por $C$. albicans que los ratones wild-type (40). Saijo et al. (41) registraron resultados similares en la protección de ratones contra P. carinii.

Actualmente se producen 2 formas de $\beta$-glucanos de $S$. cerevisiae: una forma particular insoluble (Whole Glucan Particle - WGP) y otra soluble (PGG-glucano). El WGP se obtiene por purificación a partir de paredes celulares deshidratadas de levaduras después de la extracción de las proteínas celulares, ácidos nucleicos, lípidos y oligosacáridos (quitinas y mananos) (figura 2) (42); ha recibido el nombre común de zymosan (29, 43). La empresa Biothera (USA) comercializa WellmuneÒ (WGP) como BetaRight B-WGP (70\% $\beta$-glucanos) y como Wellmune Dispersible WGP-D (75\% $\beta$-glucanos); también existe una forma soluble, Wellmune WGP-S (75\% $\beta$-glucanos). EI PGG, Ilamado betafectina (poli-1-6-beta-D-glucopiranosil1-3-beta-D-glucopiranosa) es un polímero de glucosa altamente purificado y ramificado obtenido por la hidrólisis ácida del WGP (44). Ambas formas de $\beta$-glucanos han sido evaluadas en estudios pre-clínicos y clínicos.

Un estudio in vitro mostró que la incubación de sangre humana con $\beta$-glucanos solubles de $S$. cerevisiae a concentraciones de 2 a $20 \mu \mathrm{g} / \mathrm{mL}$ aumentaba significativamente la producción de TNF- $\alpha$, IL-6, IL-8 y del factor tisular monocitario (TF) (45). También se han reportado efectos antimutagénicos
$(46,47)$ quimioprotectores y anticancerígenos de estos compuestos (48-51). Hay pocos estudios realizados en humanos con el $\beta$-glucano PGG. Babineau et al. publicaron dos estudios aleatorios controlados sobre el efecto del PGG sobre el riesgo de infecciones postoperatorias en pacientes sometidos a cirugía torácica o abdominal. A estos pacientes $(n=67$ y 34$)$ se les administró $\beta$-glucanos por vía intravenosa, observándose un riesgo significativamente menor de infecciones postoperatorias, como también un menor uso de antibióticos y tiempo de permanencia en la Unidad de Cuidados Intensivos $(52,53)$.

En un estudio multi-céntrico aleatorizado realizado en 1.249 pacientes con alto riesgo de infección ó muerte por cirugía gastrointestinal, se observó que la inyección perioperatoria de 4 dosis de $\mathrm{PGG}(0,5$ o $1 \mathrm{mg} / \mathrm{kg}$ de peso) reducía significativamente en un $39 \%$ las infecciones post-operatorias y mejoraría la sobrevida, principalmente de los sujetos malnutridos, comparado con el grupo placebo. Sin embargo, el experimento clínico debió ser interrumpido antes de tiempo, debido a efectos adversos más frecuentes en el grupo tratado con PGG (54).

Últimamente se han realizado ensayos clínicos con WGP, observándose una reducción de los episodios de alergia y de infecciones del tracto respiratorio. Uno de ellos evaluó durante la estación de invierno la respuesta inmune de sujetos sanos después de administrar por 90 días 500 mg/día de WGP, comparado con $500 \mathrm{mg} /$ día de harina de arroz en el grupo placebo. No se observaron diferencias significativas en la incidencia de infección respiratoria entre ambos grupos; sin embargo en el grupo experimental se redujo la frecuencia de fiebre además del ausentismo laboral a causa de los resfriados

\section{FIGURA 2}

Estructura de WGP® 3-6 $\beta$-D-glucano. Durante su purificación se eliminan de las paredes celulares de la levadura: manoproteínas y contenidos celulares internos.

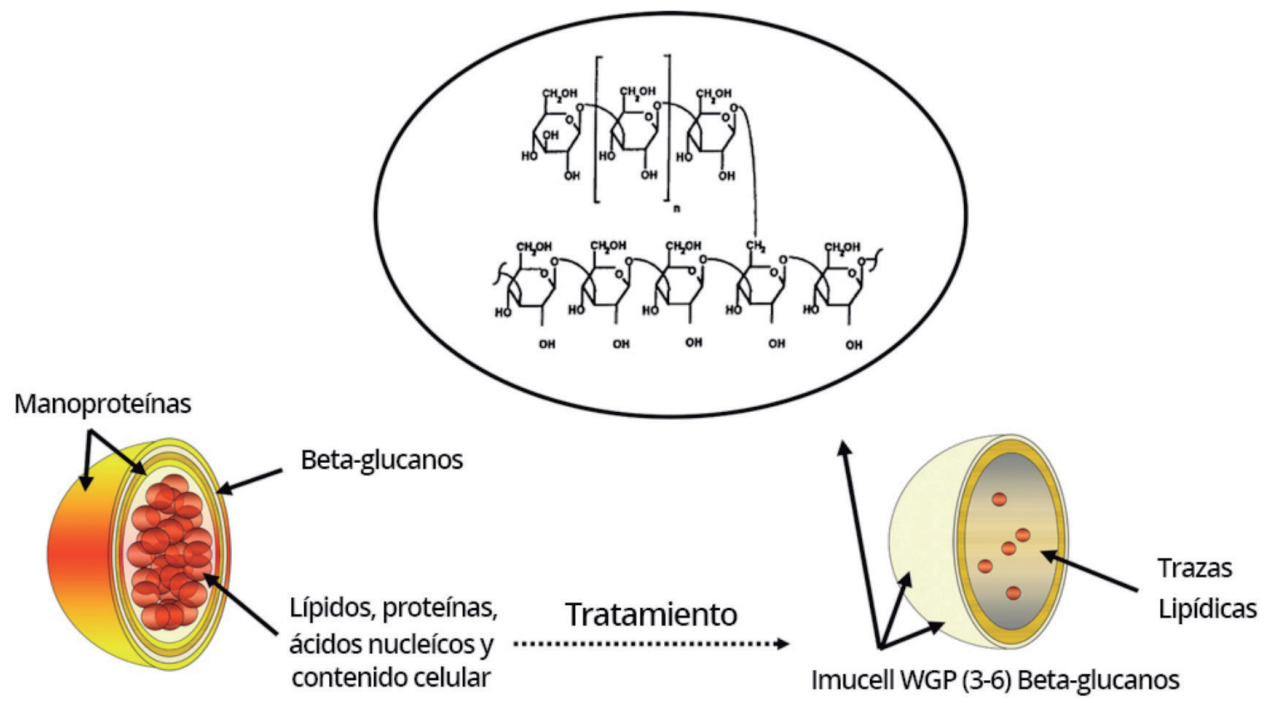

Adaptado y autorizado de: Babíček K, Čechová I, Simon RR, Harwood M, Cox DJ. Toxicological assessment of a particulate yeast (1,3/1,6)- $\beta$-d-glucan in rats.

Food Chem Toxicol. 2007; 45(9):1719-30. 
$(0,00 \pm 0,00$ vs $1,38 \pm 1,25$ días, $p=0,026)(55)$. Un ensayo controlado y doble ciego realizado en 75 maratonistas mostró que el consumo 250 y 500 mg/día de WGP durante 4 semanas post-maratón reducía significativamente la frecuencia de infecciones del tracto respiratorio. Solo $8 \%$ del grupo tratado reportó infecciones respiratorias frente a un $24 \%$ en el grupo placebo, mejorándose además, la sensación de fatiga, tensión e incrementando el vigor de los sujetos tratados (56). En otro estudio se observó que la suplementación con $250 \mathrm{mg} /$ día de WGP durante 4 semanas redujo significativamente en $28 \%$ los síntomas generales de alergia al polen de ambrosia y en $52 \%$ su intensidad; sin embargo, no tuvo ningún efecto en los niveles séricos de IgE. Por otra parte, la suplementación con WGP mejoró en 19\% el estado físico general de los sujetos y en un $7 \%$ su bienestar emocional, en comparación con el grupo control (57).

La actividad anti-alérgica de los $\beta$-glucanos también ha sido evaluada en estudios clínicos epidemiológicos y en estudios in vitro (58). En un estudio en doble ciego y controlado por placebo realizado en 24 pacientes con rinitis alérgica, el uso de $\beta$-glucanos por 12 semanas disminuyó las concentraciones de IL-4 e IL-5 en el fluido nasal sin cambios en el grupo placebo (59). El suplemento de $\beta$-glucanos por 10 días durante y post ejercicio aumentó el potencial de los leucocitos sanguíneos para producir IL-2, IL-4, IL-5 e IFN-Y, sugiriendo que los $\beta$-glucanos son capaces de modular la inmunidad después del ejercicio (60). Sólo un estudio realizado con ratones Balb/c comparó los 2 tipos de $\beta$-glucanos PGG y WGP. A los animales se les inyectó por vía subcutánea una dosis única de WGP $(200 \mu g)$ o de PGG $(50 \mu g) 7$ días antes de la inoculación de $B$. anthracis. El tratamiento con ambos $\beta$-glucanos disminuyó la carga bacteriana en los pulmones e incrementó significativamente la sobrevida de los ratones tanto en el grupo tratado con PGG $(86,4 \%, p=0.00005)$ como en el grupo tratado con WGP (81.8\%, $p=0.00024)$, en comparación del grupo control donde la sobrevida fue del 31,8\% (43).

\section{INOCUIDAD DE LOS $\beta$-GLUCANOS DE LEVADURA}

Sólo un estudio de toxicidad aguda y sub-crónica de $\beta$-glucanos de levadura (WGP) se ha desarrollado en ratas, cuyos resultados muestran la ausencia de efectos negativos en el peso de los animales y en la incidencia de morbi-mortalidad luego de haber administrado $2 \mathrm{~g} / \mathrm{kg}$ de peso corporal de $\beta$-glucanos por 14 días (61). Su inocuidad ha sido reconocida como Generally Recognized as Safe (producto "GRAS") por la FDA (Food and Drug Administration) de EE.UU. en 2007, considerando que $S$ cerevisiae esta naturalmente presente en la dieta humana (en particular para el proceso de panificación) desde el primer año de vida (62). La Agencia Europea de Inocuidad Alimentaria (EFSA), menciona que los datos de toxicidad aguda, sub-crónica y crónica son limitados en humanos, pero que esto no es realmente un motivo de preocupación debido a que el consumo de S. cerevisiae es habitual a través de los productos panaderos y de cerveza artesanal y que, además, no representa un mayor riesgo de alergia. Considerando estos antecedentes y en forma similar a la FDA, la EFSA concluyó que el consumo de $\beta$-glucanos de levadura es seguro para el ser humano (63).

\section{USOS DE $\beta$-GLUCANOS EN LA INDUSTRIA}

\section{ALIMENTARIA Y REGULACIONES LEGALES}

En la industria alimentaria, los $\beta$-glucanos de cebada y avena se utilizan como aditivos para la elaboración de panes $(21,64)$ y pastas $(20,65)$ debido a que mejoran la estabilidad, la resistencia a la deformación, la elasticidad de las masas así como su volumen, permitiendo retener la humedad y remplazar el uso del azúcar (66). Últimamente su utilización se ha extendido al desarrollo de helados bajos en grasa y yogures (2). Como se detalló anteriormente, tanto la FDA como la EFSA han autorizado mensajes de salud (health claims) que declaran que el consumo diario de $3 \mathrm{~g}$ de $\beta$-glucanos de avena o cebada puede reducir los niveles de colesterol y el riesgo de enfermedad cardiovascular $(8,67-69)$. Es muy probable que esta autorización de parte de las instancias reguladoras estimule el desarrollo de nuevos alimentos funcionales incorporando $\beta$-glucanos de avena o cebada.

En cuanto a los $\beta$-glucanos de levadura, la declaración de inocuidad emitida por la FDA y la EFSA permite incluirlos en una variedad de alimentos incluyendo bebidas destinados a la población en general. Estos ingredientes están presentes en el mercado internacional principalmente bajo las marcas Yestimun $₫$, Wellmune $₫$ y Biolex $₫$ y recientemente, en 2011, la EFSA autorizó el uso de 3 formas de $\beta$-glucanos de levadura (BetaRight WGP, Wellmune WGP Dispersible y Wellmune WGP soluble) como ingredientes alimenticios inocuos. La empresa Biothera ha incorporado sus $\beta$-glucanos de levadura (Wellmune (B) a productos horneados, jugos, cereales, lácteos, derivados de plantas y sopas con concentraciones aproximadas de 200 mg por porción de consumo; también lo comercializa como suplemento alimenticio.

Sin embargo, cabe destacar que tanto la FDA como la EFSA han rechazado las solicitudes de mensajes de salud realizadas por las empresas en relación con Yestimun $®$, respuesta inmune y protección frente a patógenos del tracto respiratorio superior, basado en el bajo número de ensayos en humanos presentado, y la insuficiencia de la evidencia científica obtenida de los estudios in vitro y en animales $(70,71)$

\section{CONCLUSIÓN}

Los $\beta$-glucanos tienen un efecto fisiológico dependiendo de su fuente de origen. En el caso de los cereales son un componente importante en los alimentos en la modulación de desregulaciones metabólicas asociadas al síndrome metabólico. Sin embargo los distintos efectos dependerán de: la dosis, su estructura, peso molecular y el tipo de alimento. Los efectos fisiológicos de los $\beta$-glucanos de cereales se atribuyen principalmente a sus características fisicoquímicas y estructurales que interactúan con el tracto gastrointestinal, reflejándose en su capacidad de generar soluciones viscosas en el intestino delgado y fermentación en el colon. Por otra parte en los $\beta$-glucanos de levaduras y hongos se ha observado efectos positivos sobre la inmunidad del huésped, previniendo los riesgos de infecciones o cáncer. Sin embargo los datos no resultan ser del todo concluyentes, pues aún no se sabe cómo será su respuesta a largo plazo.

\section{RESUMEN}

Los $\beta$-glucanos son polímeros de glucosa (polisacáridos) de elevado peso molecular que se encuentran en forma natural en la pared celular de diversos organismos vivos como bacterias, levaduras, hongos y plantas (cereales como avena y cebada). Los $\beta$-glucanos son inocuos y actualmente son utilizados por las empresas de alimentos como agentes texturizantes. Además, antecedentes científicos sugieren que, dependiendo de su estructura fisicoquímica y de su origen, su consumo se asociaría a efectos beneficiosos para la salud del ser humano como la disminución de la concentración plasmática de colesterol total y la reducción del índice glicémico de los 
alimentos que lo incluyen. Un mensaje de salud sobre consumo de $\beta$-glucanos de cereales y disminución del colesterol ha sido aceptado tanto por la FDA de EE.UU. como por la EFSA de la U.E. Por esta razón los $\beta$-glucanos están siendo utilizados cada vez más para el desarrollo de alimentos funcionales en varios países del mundo. Sin embargo, el efecto de los $\beta$-glucanos de levadura sobre la estimulación de la respuesta inmune aún está en discusión.

Palabras clave: $\beta$-glucanos, función inmune, resistencia a infecciones, colesterol sanguíneo, glucosa en sangre.

\section{BIBLIOGRAFÍA}

1. Volman JJ, Mensink RP, Ramakers JD, de Winther MP, Carlsen $H$, Blomhoff $R$, et al. Dietary $(1 \rightarrow 3),(1 \rightarrow 4)-\beta-d-$ glucans from oat activate nuclear factor- $\kappa B$ in intestinal leukocytes and enterocytes from mice. Nutr Res. 2010;30(1):40-8.

2. Brennan CS, Cleary LJ. The potential use of cereal $(1 \rightarrow 3,1 \rightarrow 4)-\beta-d$-glucans as functional food ingredients. J Cereal Sci. 2005;42(1):1-13.

3. Stuart IM, Loi L, Fincher GB. Immunological comparison of $(1 \rightarrow 3,1 \rightarrow 4)$ - $\beta$-glucan endohydrolases in germinating cereals. J Cereal Sci. 1987;6(1):45-52.

4. A. Bacic GBF, B. A. Stone. Chemistry, Biochemistry, and Biology of (1-3)-[beta]-Glucans and Related Polysaccharides. Academic Press, Amsterdam, The Netherlands. 2009.

5. Teas J. The dietary intake of laminaria, a brown seaweed, and breast cancer prevention. Nutr Cancer. 1982;4(3):21722.

6. Weis AL, Wasser SP. Therapeutic Effects of Substances Occurring in Higher Basidiomycetes Mushrooms: A Modern Perspective. 1999;19(1):32.

7. Lazaridou A, Biliaderis CG. Molecular aspects of cereal $\beta$-glucan functionality: Physical properties, technological applications and physiological effects. J Cereal Sci. 2007;46(2):101-18.

8. Wood PJ. Cereal $\beta$-glucans in diet and health. J Cereal Sci. 2007;46(3):230-8.

9. Butt M, Tahir-Nadeem M, Khan M, Shabir R, Butt M. Oat: unique among the cereals. Eur J Nutr. 2008;47(2):68-79.

10. Kim HS, Hong JT, Kim Y, Han S-B. Stimulatory Effect of $\beta$-glucans on Immune Cells. Immune Netw. 2011;11(4):191-5.

11. $\neq$ Dikeman CL, Fahey GC. Viscosity as Related to Dietary Fiber: A Review. Critical Rev Food Sci Nutr. 2006;46(8):64963.

12. Wood PJ, Weisz J, Blackwell B. Structural Studies of (1-3) (1-4)-beta-D-Glucans by 13C-nuclear magnetic-resonance spectroscopy and by rapid analysis of cellulose-like regions using high-performance anion-exchange chromatography of oligosaccharides released by lichenase. Cereal Chem. 1994;71(3):301-7.

13. Tiwari U, Cummins E. Meta-analysis of the effect of $\beta$-glucan intake on blood cholesterol and glucose levels. Nutr. 2011;27(10):1008-16.

14. Lovegrove JA, Clohessy A, Milon H, Williams CM. Modest doses of $\beta$-glucan do not reduce concentrations of potentially atherogenic lipoproteins. Am J Clin Nutr. 2000;72(1):49-55.

15. Karmally W, Montez MG, Palmas W, Martinez W, Branstetter A, Ramakrishnan R, et al. Cholesterol-Lowering Benefits of Oat-Containing Cereal in Hispanic Americans. J Am Diet Assoc. 2005;105(6):967-70.

16. Kerckhoffs DA, Hornstra G, Mensink RP. Cholesterol- lowering effect of $\beta$-glucan from oat bran in mildly hypercholesterolemic subjects may decrease when $\beta$-glucan is incorporated into bread and cookies. Am J Clin Nutr. 2003;78(2):221-7.

17. Ripsin CM, Keenan JM, R. JD. Oat products and lipid lowering: A meta-analysis. JAMA. 1992;267(24):3317-25.

18. EFSA Panel on Dietetic Products NaAN. Scientific Opinion on the substantiation of a health claim related to oat beta-glucan and lowering blood cholesterol and reduced risk of (coronary) heart disease pursuant to Article 14 of Regulation (EC) No 1924/2006. EFSA J 2010;8(12):1885.

19. Granfeldt $Y$, Nyberg L, Bjorck I. Muesli with $4 \mathrm{~g}$ oat beta-glucans lowers glucose and insulin responses after a bread meal in healthy subjects. European J Clin Nutr. 2007;62(5):600-7.

20. Chillo S, Ranawana DV, Pratt M, Henry CJ. Glycemic response and glycemic index of semolina spaghetti enriched with barley beta-glucan. Nutrition. 2011;27(6):653-8. Epub 2010/09/28.

21. Thondre PS, Henry CJ. High-molecular-weight barley beta-glucan in chapatis (unleavened Indian flatbread) lowers glycemic index. Nutr Res. 2009;29(7):480-6. Epub 2009/08/25.

22. Jaskari J, Kontula $P$, Siitonen A, Jousimies-Somer $H$, Mattila-Sandholm T, Poutanen K. Oat beta-glucan and xylan hydrolysates as selective substrates for Bifidobacterium and Lactobacillus strains. Appl Microbiol Biotechnol. 1998;49(2):175-81. Epub 1998/04/16.

23. Kontula $P$, Jaskari J, Nollet L, De Smet I, von Wright $A$, Poutanen $K$, et al. The colonization of a simulator of the human intestinal microbial ecosystem by a probiotic strain fed on a fermented oat bran product: effects on the gastrointestinal microbiota. Appl Microbiol Biotechnol. 1998;50(2):246-52. Epub 1998/10/09.

24. Dongowski G, Huth M, Gebhardt E, Flamme W. Dietary fiber-rich barley products beneficially affect the intestinal tract of rats. J Nutr. 2002;132(12):3704-14. Epub 2002/12/07.

25. Drzikova B, Dongowski G, Gebhardt E. Dietary fibre-rich oat-based products affect serum lipids, microbiota, formation of short-chain fatty acids and steroids in rats. British J Nutr. 2005;94(6):1012-25. Epub 2005/12/15.

26. Snart J, Bibiloni $R$, Grayson T, Lay C, Zhang $H$, Allison $G E$, et al. Supplementation of the diet with high-viscosity beta-glucan results in enrichment for lactobacilli in the rat cecum. Applied Environm Microbiol. 2006;72(3):1925-31. Epub 2006/03/07.

27. Mitsou EK, Panopoulou N, Turunen K, Spiliotis V, Kyriacou $A$. Prebiotic potential of barley derived $\beta$-glucan at low intake levels: A randomised, double-blinded, placebo-controlled clinical study. Food Res Internat. 2010;43(4):1086-92.

28. Turunen K, Tsouvelakidou E, Nomikos T, Mountzouris KC, Karamanolis D, Triantafillidis J, et al. Impact of beta-glucan on the faecal microbiota of polypectomized patients: A pilot study. Anaerobe. 2011;17(6):403-6.

29. Volman JJ, Ramakers JD, Plat J. Dietary modulation of immune function by $\beta$-glucans. Physiology Amp Behavior. 2008;94(2):276-84.

30. Wang $Y$, Zhang L, Li Y, Hou X, Zeng F. Correlation of structure to antitumor activities of five derivatives of a $\beta$-glucan from Poria cocos sclerotium. Carbohydrate Res. 2004;339(15):2567-74.

31. Xu X, Yasuda M, Mizuno M, Ashida $H$. $\beta$-Glucan from Sac- 
charomyces cerevisiae reduces lipopolysaccharide-induced inflammatory responses in RAW264.7 macrophages. Biochimica et Biophysica Acta (BBA) - General Subjects. 2012;1820(10):1656-63.

32. Harada K, Itashiki Y, Takenawa T, Ueyama Y. Effects of Lentinan alone and in combination with fluoropyrimidine anticancer agent on growth of human oral squamous cell carcinoma in vitro and in vivo. Internat $J$ Oncol. 2010;37(3):623-31.

33. Brown GD. Dectin-1: a signalling non-TLR pattern-recognition receptor. Nat Rev Immunol. 2006;6:33-43.

34. Călugăru A, Cremer L, Lupu AR, Bădulescu MM, Apetrei NS, Moscovici M, et al. Recognition and modulation of Dectin-1 and TLR-2 receptors by curdlan derivatives and purified natural extracts. Roum Arch Microbiol Immunol. 2009;68.(3):119-24.

35. Zhou L-d, Zhang Q-h, Zhang Y, Liu J, Cao Y-m. The shiitake mushroom-derived immuno-stimulant lentinan protects against murine malaria blood-stage infection by evoking adaptive immune-responses. Internat Immunopharmacol. 2009;9(4):455-62.

36. Saegusa S TM, Kaminogawa S, Hosoi T. Candida albicans and Saccharomyces cerevisiae induce interleukin-8 production from intestinal epithelial-like Caco-2 cells in the presence of butyric acid. FEMS Immunol Med Microbiol. 2004; 41:227-35.

37. Taylor PR, Brown GD, Reid DM, Willment JA, L. M-P, Gordon $S$, et al. The beta-glucan receptor, dectin-1, is predominantly expressed on the surface of cells of the monocyte/macrophage and neutrophil lineages. J Immunol 2002;169:3876-82

38. Goodridge HS, Wolf AJ, Underhill DM. Beta-glucan recognition by the innate immune system. Immunol Rev. 2009;230 38-50.

39. Lebron F, Vassallo $R$, Puri V, Limper AH. Pneumocystis carinii cell wall beta-glucans initiate macrophage inflammatory responses through NF-kappaB activation. J Biol Chem. 2003;278:25001-8.

40. Taylor PR TS, Willment JA, Dennehy KM, Rosas M, Findon $H$. Dectin-1 is required for beta-glucan recognition and control of fungal infection. . Nat Immunol 2007;8:31-8.

41. Saijo S FN, Furuta T, Chung SH, Kotaki H, Seki K. Dectin-1 is required for host defense against Pneumocystis carinii but not against Candida albicans. Nat Immunol. 2007;8:39-46.

42. Jamas S ED, Ostroff $G$. . Method for immune system activation by administration of a beta(1-3) glucan which is produced by Saccharomyces cerevisiae strain R4. US Patent 5,504,079. 1996.

43. Kournikakis B, Mandeville R, Brousseau P, Ostroff $G$. Anthrax-protective effects of yeast beta 1,3 glucans. Med Gen Med. 2003;5(1).

44. Jamas S EDJ, Ostroff GR, Onderdonk AB. PGG -- a novel class of macrophage activating immunomodulators. Polymer. 1990;31:194-5.

45. Engstad CS, Engstad RE, Olsen J-O, Østerud B. The effect of soluble $\beta-1,3-$ glucan and lipopolysaccharide on cytokine production and coagulation activation in whole blood. Internat Immunopharmacol. 2002;2(11):1585-97.

46. Miadoková E, Svidová S, Vlčková V, Dúhová V, Nad'ová $S$, Rauko $P$, et al. Diverse biomodulatory effects of glucomannan from Candida utilis. Toxicol in Vitro. 2006; 20(5): 649-57.

47. Xue Bin Z, Ohta Y. Antimutagenicity of cell fractions of microorganisms on potent mutagenic pyrolysates. Mutation
Res/Genetic Toxicol. 1993;298(4):247-53.

48. Cheung N-K, Modak S, Vickers A, Knuckles B. Orally administered $\beta$-glucans enhance anti-tumor effects of monoclonal antibodies. Cancer Immunol Immunother. 2002;51(10):557-64.

49. Chorvatovičová D, Navarová J. Suppressing effects of g/ucan on micronuclei induced by cyclophosphamide in mice. Mutation Res Lett. 1992;282(3):147-50.

50. Hong F, Yan J, Baran JT, Allendorf DJ, Hansen RD, Ostroff $G R$, et al. Mechanism by Which Orally Administered $\beta-1,3-$ Glucans Enhance the Tumoricidal Activity of Antitumor Monoclonal Antibodies in Murine Tumor Models. J Immunol. 2004;173(2):797-806.

51. Yeun-hwa Gu YT, Takashi Nakamura, Takeo Hasegawa, Ikukatsu Suzuki, Masami Oshima, Hitoshi Tawaraya, Yoshimi Niwano. Enhancement of Radioprotection and Anti-Tumor Immunity by Yeast-Derived $\beta$-Glucan in Mice. J Medic Food. 2005;8(2):154-8.

52. Babineau TJ, Hackford A, Kenler A. A phase II multicenter, double-blind, randomized, placebo-controlled study of three dosages of an immunomodulator (PGG-glucan) in high-risk surgical patients. Arch Surg. 1994;129(11):120410.

53. Babineau TJ, Marcello P, Swails W, Kenler A, Bistrian B, Forse RA. Randomized phase I/II trial of a macrophagespecific immunomodulator (PGG-glucan) in high-risk surgical patients. Ann Surgical Oncol. 1994;220:601-9.

54. Dellinger E BTJBP. Effect of PGG-glucan on the rate of serious postoperative infection or death observed after high-risk gastrointestinal operations. Arch Surgery. 1999;134(9):977-83.

55. Feldman S, H. I. Schwartz, D. S. Kalman, A. Mayers, H. M. Kohrman, R. Clemens and D. R. Krieger. Randomized phase II clinical trials of Wellmune WGP® for immune support during cold and flu season. J Applied Res. 2009;9:30-42.

56. Talbott S, Talbott J. Effect of BETA 1, 3/1, 6 GLUCAN on Upper Respiratory Tract Infection Symptoms and Mood State in Marathon Athletes. J Sports Sci Med. 2009;8:509-15.

57. Talbott SM, Talbott JA, Talbott TL, Dingler E. B-Glucan supplementation, allergy symptoms, and quality of life in self-described ragweed allergy sufferers. Food Science Nutr. 2013;1(1):90-101.

58. Jesenak M, Majtan J, Rennerova Z, Kyselovic J, Banovcin $P$, Hrubisko M. Immunomodulatory effect of pleuran ( $\beta$-glucan from Pleurotus ostreatus) in children with recurrent respiratory tract infections. Internat Immunopharmacol. 2013;15(2):395-9.

59. Cengiz Kirmaz, Papatya Bayrak, Ozge Yilmaz, Hasan Yuksel , Bayar C. Effects of glucan treatment on the Th1/Th2 balance in patients with allergic rhinitis: a double-blind placebo-controlled study. European Cytokine Network. 2005;16(2):128-34.

60. Carpenter KC, Breslin WL, Davidson T, Adams A, McFarlin $B K$. Baker's yeast $\beta$-glucan supplementation increases monocytes and cytokines post-exercise: implications for infection risk? British J Nutr. 2013;109(03):478-86.

61. Babíček K, Čechová I, Simon RR, Harwood M, Cox $D J$. Toxicological assessment of a particulate yeast $(1,3 / 1,6)-\beta$-d-glucan in rats. Food Chem Toxicol. 2007; 45(9): 1719-30.

62. FAO/WHO. Biotechnology y Food Safety. Report of a Joint FAO/WHO Consultation. Rome, Food y Agriculture Organization of the United Nations (FAO)/World Health Organization (WHO); Geneva, Switz., FAO Food and 
Nutrition.1996.

63. EFSA. EFSA Panel on Dietetic Products, Nutrition and Allergies (NDA); Scientific Opinion on the safety of "Yeast beta-glucans" as a Novel Food ingredient. EFSA J 2011;9 (5):2137-59.

64. Cavallero A, Empilli S, Brighenti F, Stanca AM. High $(13,14)$-beta-Glucan Barley Fractions in Bread Making and their Effects on Human Glycemic Response. J Cereal Sci. 2002;36(1):59-66.

65. Chillo S, Ranawana DV, Henry CJK. Effect of two barley $\beta$-glucan concentrates on in vitro glycaemic impact and cooking quality of spaghetti. LWT - Food Sci Technol. 2011;44(4):940-8.

66. Skendi A, Biliaderis CG, Papageorgiou M, Izydorczyk MS. Effects of two barley $\beta$-glucan isolates on wheat flour dough and bread properties. Food Chem. 2010;119(3):1159-67.

67. FDA. Food labelling: Health claims; Oats and coronary heart disease; Final rule. Federal Register. 1997;62(15):3583-
601.

68. FDA. Food labeling, health claims; soluble dietary fiber from certain foods and coronary heart disease. Federal Register 21 CFR Part 101. 2005;70(246):76150-62.

69. FDA. Food labelling: Specific requirements for health claims; Health claims: Soluble fiber from certain foods and risk of coronary heart disease (CHD). 21 Code of Federal Regulations. 2008;2:142-6.

70. EFSA. EFSA Panel on Dietetic Products, Nutrition and Allergies (NDA); Scientific Opinion on the substantiation of a health claim related to Yestimun $₫$ and immune responses pursuant to Article 13(5) of Regulation (EC) No 1924/2006. EFSA J. 2010;8(5): 1607.

71. EFSA Panel on Dietetic Products NaAN. Scientific Opinion on the substantiation of a health claim related to Yesti-

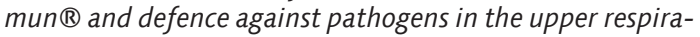
tory tract pursuant to Article 13(5) of Regulation (EC) No 1924/20061. EFSA J 2013;11(4):3159. 